\title{
Does addition of craving management tools in a stop smoking app improve quit rates among adult smokers? Results from BupaQuit pragmatic pilot randomised controlled trial
}

Digital Health

Volume 7: 1-13

(C) The Author(s) 2021

Article reuse guidelines:

sagepub.com/journals-permissions

DOI: $10.1177 / 20552076211058935$

journals.sagepub.com/home/dhj

(S)AGE

\author{
Aleksandra Herbec ${ }^{1,2,3}$ (D), Lion Shahab ${ }^{1,3}$, Jamie Brown ${ }^{1,3,4}$, Harveen \\ Kaur Ubhi ${ }^{1,3}$, Emma Beard ${ }^{1,3,4}$, Alexandru Matei ${ }^{5,6}$ and Robert West ${ }^{1,3}$
}

\section{Abstract}

Objectives: Delivery of craving management tools via smartphone applications (apps) may improve smoking cessation rates, but research on such programmes remains limited, especially in real-world settings. This study evaluated the effectiveness of adding craving management tools in a cessation app (BupaQuit).

Methods: The study was a two-arm pragmatic pilot parallel randomised controlled trial, comparing a fully-automated BupaQuit app with craving management tool with a control app version without craving management tool. A total of 425 adult UK-based daily smokers were enrolled through open online recruitment (February 2015-March 2016), with no researcher involvement, and individually randomised within the app to the intervention $(n=208)$ or control $(n=217)$. The primary outcome was self-reported 14-day continuous abstinence assessed at 4-week follow-up. Secondary outcomes included 6-month point-prevalence and sustained abstinence, and app usage. The primary outcome was assessed with Fisher's exact test using intent to treat with those lost to follow-up counted as smoking. Participants were not reimbursed.

Results: Re-contact rates were $50.4 \%$ at 4 weeks and $40.2 \%$ at 6 months. There was no significant difference between intervention and control arms on the primary outcome $(13.5 \%$ vs $15.7 \% ; p=0.58$; relative risk $=0.86$, $95 \%$ confidence interval $=$ $0.54-1.36)$ or secondary cessation outcomes (6-month point prevalence: $14.4 \%$ vs $17.1 \%, p=0.51$; relative risk $=0.85,95 \%$ confidence interval $=0.54-1.32$; 6 -month sustained: $11.1 \%$ vs $13.4 \%, p=0.55$; relative risk $=0.83$, $95 \%$ confidence interval $=$ $0.50-1.38)$. Bayes factors supported the null hypothesis $(B[0,0,1.0986]=0.20)$. Usage was similar across the conditions (mean/median logins: $9.6 / 4$ vs $10.5 / 5$; time spent: $401.8 / 202$ s vs $325.8 / 209$ s).

Conclusions: The addition of craving management tools did not affect cessation, and the limited engagement with the app may have contributed to this.

Keywords

smoking, cessation, smartphone, cravings, randomised controlled trial

Submission date: 20 January 2021; Acceptance date: 22 October 2021

\section{Introduction}

Use of face-to-face and telephone-based smoking cessation support is low even when it is free at the point of access. ${ }^{1}$ Smartphone apps may appeal to smokers not willing to use these forms of support. ${ }^{2}$ In 2016 , over $85 \%$ of the UK population had access to a smartphone. ${ }^{3}$ However, most cessation apps do not offer evidence-based support. ${ }^{4-7}$ Results of randomised controlled trials (RCTs) ${ }^{8-10}$ and observational

\footnotetext{
${ }^{1}$ Department of Behavioural Science and Health, University College London, UK ${ }^{2}$ Department of Clinical, Educational and Health Psychology, UCL Centre for Behaviour Change, University College London, UK

${ }^{3}$ UCL Tobacco and Alcohol Research Group (UTARG), University College London, UK ${ }^{4}$ Department of Clinical, Educational and Health Psychology, University

College London, UK

${ }^{5}$ Bupa Centre Medical, UK

${ }^{6}$ Department of Computer Science, University College London, UK
}

Corresponding author:

Aleksandra Herbec, Department of Behavioural Science and Health, University College London, Rm 215, 1-19 Torrington Place, London WC1E 6BT, UK. Email: a.herbec@ucl.ac.uk 
studies ${ }^{11-13}$ have not yet provided clear evidence that such apps can aid cessation. Recently a decision-aid app improved self-reported quit rates over a control app version. ${ }^{14}$ The present pilot RCT assessed how far the inclusion of craving management tools (CMTs) in an app could improve cessation. Furthermore, in order to recreate for the participants a more authentic experience of app download, and to increase the generalisability of findings, the study involved limited contact with the researchers and low participant burden at enrolment, in contrast to earlier research. ${ }^{8,9,12}$

Cigarette craving can be defined as the experience of strong motivation (desire, need or urge) to smoke and is predictive of relapse. ${ }^{15}$ Several techniques reduce momentary cravings, including distraction, imagining pleasant experiences, ${ }^{16}$ relaxation, ${ }^{17}$ physical exercise ${ }^{18}$ and yogic breathing. ${ }^{19}$ Cessation interventions that include behaviour change techniques $(\mathrm{BCTs})^{20}$ that reduce, or improve coping, with cravings appear to improve success rates. ${ }^{20}$ An advantage of apps, also over SMS texting, is that they could be used online and offline, present multimedia content, and thus offer an opportunity to deliver or prompt use of different CMTs when appropriate. In principle, such apps could improve quit rates.

The SF28 ('SmokeFree28') app is an existing app that supports smokers to be smoke-free for 28 days as the first step to long-term abstinence, through offering features to set up a quit date and monitor progress, as well as advice on cessation and medication use, and motivational content (http://www.sf28.co.uk/). ${ }^{11,21}$ SF28 is informed by PRIME theory of motivation, which postulates that quitting requires maintaining sufficiently high desire and capacity to override emerging impulses to smoke. ${ }^{22}$ In an observational study, SF28 produced self-reported short-term abstinence that was higher than would have been expected with unaided cessation. ${ }^{11}$ This app was also judged to contain all the evidencebased features that might be expected to improve cessation ${ }^{23}$ and achieved relatively high engagement rates $(8.5$ ( $\mathrm{SD}=$ 9.0) mean logins). ${ }^{11}$ SF28 was therefore chosen as the basis for the development of a new app to evaluated specific CMTs. The new app was sponsored by the healthcare company Bupa and was called 'BupaQuit'. The appearance of SF28 was redesigned while keeping the key logic, content, and the user flow was similar.

Two versions of BupaQuit were created: one with a set of CMTs, and the other without them. The control app was designed to be a minimal credible intervention (MCI), ${ }^{24}$ and to be similar to the intervention app in many respects (e.g. user journey, layout, core advice), but not to offer key intervention components. Provision of a true inactive control condition or a waitlist in smartphone research may be impossible given the availability of other free quit smoking apps, and so the control version of BupaQuit was judged to be fairer and a more realistic comparison for the trial. Using an MCI would also support enrolment and follow-up, and ensure comparable user experience and data collection across study arms. Furthermore, a poorly performing control app could lack credibility, damage the long-term reputation of BupaQuit program, undermine cessation rather than simply being 'neutral' and encourage users to seek alternative apps. ${ }^{24}$

\section{Aims}

This study aimed to estimate the impact of the inclusion of CMTs within an app that was live on app stores on cessation and engagement levels, compared with an app version without those features, and to assess the feasibility of remote enrolment and follow-up procedures.

\section{Materials and methods}

\section{Design}

This study was a two-arm parallel double-blind pragmatic pilot RCT conducted remotely in the UK, with participants randomised automatically within the app (after registration) in 1:1 ratio to either the intervention or control app (random numbers generated using a standard JavaScript library). The study was prospectively registered on an international trial registry (ISRCTN10548241). Additional documentation is available on the Open Science Framework (OSF, https:// osf.io/ge6vh/). The protocol was amended before data were unblinded (for details see Box A.1 in the Supplementary Materials).

\section{Participants recruitment}

Participants were enrolled between 18th February 2015 and 16th March 2016 through open and remote online recruitment, ${ }^{14}$ with no researcher involvement and minimal participant burden. The study was advertised through paid advertisements on Twitter and Facebook, supplemented by emails and posters within Bupa and the University. Participants were invited to a study conducted in collaboration between the University team and Bupa comparing different features within the BupaQuit app. The differences between conditions were not disclosed. The app could also be found through online searches and on UK app stores. Interested participants were directed to the project website (Figure A.1), with a study information sheet that was also available upon app download, and then to download the app for free.

\section{Participant eligibility}

Participants were eligible if they were (a) UK-based, (b) 18 years or older, (c) smoked daily, (d) wanted to make a serious quit attempt, (e) completed registration, (f) were willing to set a quit date within 2 weeks of registration, $(\mathrm{g})$ agreed to follow-up, (h) agreed to, if invited, confirm 
abstinent with a personal $\mathrm{CO}$ monitor posted to them for free, (i) consented and agreed to Bupa's End User License Agreement (EULA). Criteria (a)-(e) were assessed through a baseline questionnaire. Criteria (f)-(i) were part of consent and app onboarding. Eligibility screening was automated but later supplemented by manual checks based on the unique device ID, name and contact details (24/32, or $75 \%$ of duplicate accounts were identified manually).

\section{Sample size}

Given limited information, the effect size estimates were based on SF28 results, ${ }^{11}$ assuming that the control app would be slightly less effective and the intervention app slightly more effective, with predicted success rates of $17 \%$ and $25 \%$, respectively $(\mathrm{OR}=1.6)$. This expected difference would be clinically meaningful. ${ }^{25}$ A sample size of 812 would be required to detect this effect in two-tailed analysis with alpha $=0.05$ and $80 \%$ power. Due to slower recruitment than anticipated, and thus under-recruitment within the time and resources available, the final study sample was 425 participants, which had $51 \%$ power to detect the predicted effect. We addressed this limitation by calculating Bayes factors for the abstinence outcomes (see Data Analysis below). ${ }^{26}$

\section{Bupaquit platform}

BupaQuit was developed for $\mathrm{iOS}$ and Android by Bupa (www.bupa.com), with the process overseen primarily by the first and sixth author (for details see Box A.2). This involved adapting the original SF28 content, ${ }^{11}$ creating new content and designs to reflect Bupa branding, adding Bupa and University logos and developing a bespoke database. The control version of BupaQuit was developed simultaneously to act as a minimum credible intervention, proving basic functionality that users could expect from a cessation app. The quit plan, and look and feel of the control and intervention versions were identical. The Supplemental Materials provide BupaQuit screenshots (Figure A.2), comparison of SF28, BupaQuit intervention and control on functionality and BCTs ${ }^{27}$ (Table A.1), participant journey through the trial (Figure A.3) and app (Figure A.4). BupaQuit was accessible offline, except for changing the quit date and completing follow-up questionnaires to enable data synchronisation. Participants were free to use the app ad libitum, but the app encouraged regular (daily) use through push notifications. Due to study protocol and policy changes in iTunes store on data collection within the apps, no changes or bug fixes to BupaQuit could be made during the trial, except for increasing the size of the control app to match that of the intervention app to minimise differences on download (implemented after 196 users were enrolled).
Bupaquit control app. The control app required setting a quit date within 2 weeks of app download, encouraged use of cessation medications, offered minimal support for up to 6 weeks (14 days before the quit date: pre-quit, and up to 28 days after the quit date: post-quit), including advice on pharmacotherapy, lifestyle changes, daily push-notifications that could be disabled, brief feedback on smoking status, sections 'about the study', 'about the app', a timeline with progress and tracking of money saved, a meter for momentary cravings (a scale from $0-4^{28}$ ), and an option to share the progress on social media.

Bupaquit intervention app. In addition to the functionality in the control app, the intervention app included CMTs that were suggested to users reporting $\geq 1$ on the craving meter during post-quit app. The CMTs included components from SF28, and were informed by research or theory that suggested potential usefulness at managing cravings: a game promoting distraction, ${ }^{16,29}$ 4Weeks 2 Freedom videos presenting selfrecorded accounts of smokers trying to quit, which was designed to boost motivation and self-efficacy, ${ }^{30}$ music, audio recordings of guided relaxation routines (e.g. 'body scan'), ${ }^{31,32}$ descriptions of exercises and activities (e.g. fist clenching, brisk walking ${ }^{18,33,34}$ ), and motivation boosting tips (e.g. strengthening ex-smoker identity). ${ }^{20}$ The app also offered gamification features (e.g. unlocking of craving aids when engaging with the app), a new piece of brief advice on lifestyle changes that unlock in weeks $2-4$, and a longer feedback on smoking status. Some intervention content (e.g. videos or music) was available for free upon additional download.

\section{Trial procedures}

Figure 1 presents the flowchart of participants. After download, participants provided consent and accepted EULA (via tick box), set a quit date, registered and provided contact details, completed baseline and received access to the allocated app version. Participants meeting eligibility criteria were followed-up at 4 weeks and 6.5 months after the final quit date set during their first quit attempt (to account for 2-week grace period following the quit date $^{35}$ ). Participants were not reimbursed for their participation in the study nor for providing self-reported outcome data, which is in contrast to some previous studies. 8,10 Reimbursement or incentives were not used in the present study in order to increase external validity and generalisability of the findings to real world settings, where such reimbursement would not be offered. Moreover, as the study was conducted entirely remotely and involved behaviours that participants could have routinely engaged in outside of the study, it was judged, in agreement with the ethics committee reviewing this study, that participant burden and any levels of discomfort from participation were low and not necessitating additional incentives. 


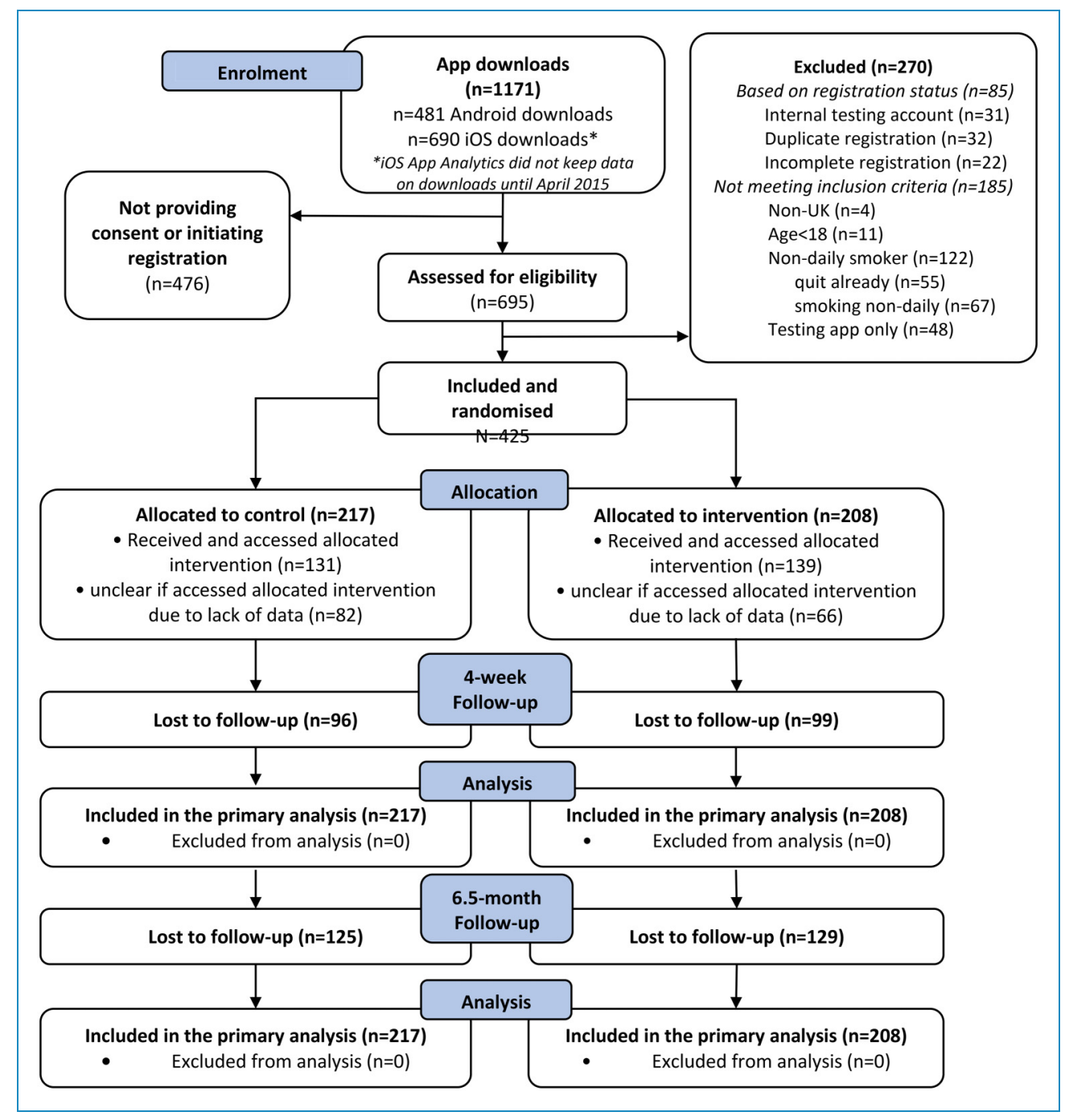

Figure 1. Flowchart of participants in the BupaQuit trial.

Early in the trial we found out that for some participants (app-data-missing) usage-related data were missing (due to database architecture, these included data on the quit date, operating system, cigarettes smoked per day, and weekly spent). The possible causes were: (i) a failure of synchronisation due to offline use (for seven users the data synchronised with a delay), (ii) interrupted installation, or (iii) not accessing the app after registration. Consequently, in the absence of quit date information, these participants were followed-up at 5 weeks and 7 months since registration. These participants were excluded in sensitivity analyses.

Tables A.2 and A.3a-b outline the schedule of procedures and questionnaires. All assessors were blind to condition allocation. At 4-weeks, the follow-up was via the app (up to three push notifications), e-mail (two emails) and phone (up to four calls). At 6.5 months, the follow-up was via email and phone. The phone follow up only asked about the smoking status. We trialled follow-up through
SMS texting, but it was not successful and was discontinued. We also attempted remote biochemical verification of abstinence using personal carbon monoxide monitors developed by Bedfont ${ }^{\circledR}$ Scientific Ltd (COmpact Smokerlyzer $\left.{ }^{\circledR}\right)$, but this proved to be an infeasible method, with only $15 \%$ of $\mathrm{CO}$ readings returned from participants reporting abstinence (the majority confirming abstinence) (for details $\operatorname{see}^{36}$ ). Possible reasons could include insufficient contact with participants and lack of reimbursement. ${ }^{8,12}$

\section{Measures}

Baseline measures. The baseline survey was mandatory, and collected data on socio-demographic characteristics; smoking, quitting, restriction on phone use and recruitment channel (Table A.2). We recorded operating system (iOS, Android, or Unkown for participants with app-missingdata), and the quit date. 
Table 1. Baseline characteristics of BupaQuit trial participants.

\begin{tabular}{|c|c|c|c|}
\hline & Total $(n=425)$ & Intervention $(n=208)$ & Control $(n=217)$ \\
\hline Age (years); Mean (SD) & $32.9(11.19)$ & $33.05(10.10)$ & $32.76(11.40)$ \\
\hline Cigarettes smoked per day; Mean (SD) & $15.32(7.17)$ & $15.08(7.32)$ & $15.58(7.03)$ \\
\hline Weekly spent on cigarettes (GBP) ${ }^{\mathrm{a}}$; Mean (SD) & $39.92(50.11)$ & $39.85(66.08)$ & $40.01(24.02)$ \\
\hline Smokes within 5 min of waking up; \% $(N)$ & $21.4(91)$ & $19.7(41)$ & $23.0(50)$ \\
\hline Confidence to stop (1-7); Mean (SD) & $4.88(1.36)$ & $4.98(1.35)$ & $4.78(1.37)$ \\
\hline Female $\%(N)$ & $45.5(193)$ & $44.7(93)$ & $46.1(100)$ \\
\hline \multicolumn{4}{|l|}{ Occupation \% $(N)$} \\
\hline Manual & $49.2(209)$ & $51.0(106)$ & $47.5(103)$ \\
\hline Non-manual & $26.4(112)$ & $25.0(50)$ & $28.6(62)$ \\
\hline Other (incl. retired, unemployed, student) & $24.5(104)$ & $25.0(52)$ & $24.0(52)$ \\
\hline Has post- 16 yrs qualification; $\%(N)$ & $68.7(29)$ & $70.2(146)$ & $67.3(146)$ \\
\hline Time with urges (0-5); Mean (SD) & $3.71(.99)$ & $2.7(.95)$ & $2.7(1.05)$ \\
\hline Strength of urges $(0-5)$; Mean (SD) & $2.81(.86)$ & $2.75(.86)$ & $2.8(.94)$ \\
\hline Made an attempt to quit last year; \% $(N)$ & $63.1(268)$ & $63.9(133)$ & $62.2(135)$ \\
\hline Stopped smoking for more than 1 week; \% $(N)$ & $76.0(323)$ & $77.9(162)$ & $74.2(161)$ \\
\hline \multicolumn{4}{|l|}{ Recruitment channel } \\
\hline Advertisement on Twitter/Facebook & $33.9(144)$ & $33.7(70)$ & $34.1(74)$ \\
\hline App store searches & $36.5(155)$ & $39.4(82)$ & $33.6(73)$ \\
\hline Other (email, word of mouth, poster) & $29.6(126)$ & $26.9(56)$ & $32.3(70)$ \\
\hline Restricted phone access during the day; \% $(N)$ & $23.3(99)$ & $22.1(46)$ & $24.4(53)$ \\
\hline \multicolumn{4}{|l|}{ Used any cessation aids in the past ${ }^{\mathrm{b}} ; \%(N)$} \\
\hline No aids & $19.1(81)$ & $18.8(39)$ & $19.4(42)$ \\
\hline Stop smoking services & $31.1(132)$ & $31.2(65)$ & $30.9(67)$ \\
\hline Medications & $52.7(224)$ & $47.6(99)$ & $57.6(125)$ \\
\hline E-cigarettes & $50.1(213)$ & $51.4(107)$ & $48.8(106)$ \\
\hline Apps & $20.2(86)$ & $21.2(44)$ & $19.4(42)$ \\
\hline Other incl. websites and quitline & $16.2(69)$ & $17.3(36)$ & $15.2(33)$ \\
\hline
\end{tabular}


Table 1. Continued.

Total $(n=425)$

Intervention $(n=208)$

Control $(n=217)$

Current use of cessation aids ${ }^{a, b} ; \%(N)$

\begin{tabular}{|c|c|c|c|}
\hline No aids & $54.2(150)$ & $59.2(84)$ & $48.9(66)$ \\
\hline Stop smoking services & 5.1. (14) & $4.9(7)$ & $5.2(7)$ \\
\hline Medications & $19.9(55)$ & $14.8(21)$ & $25.2(34)$ \\
\hline E-cigarettes & $26.0(72)$ & $24.6(35)$ & $27.4(37)$ \\
\hline Other (incl. apps, websites, quitlines) & $6.1(17)$ & $7.0(10)$ & $5.2(7)$ \\
\hline \multicolumn{4}{|l|}{ Operating system ${ }^{\mathrm{a}} ; \%(N)$} \\
\hline iOS & $36.2(154)$ & $36.1(75)$ & $36.4(79)$ \\
\hline Android & $28.9(123)$ & $32.2(67)$ & $25.8(56)$ \\
\hline Unknown & $34.7(148)$ & $31.7(66)$ & $37.8(82)$ \\
\hline Set Quit Date to Today ${ }^{a}$ & 68.9 (190) & 69.7 (99) & $67.4(91)$ \\
\hline
\end{tabular}

${ }^{a}$ Data available for 277 participants (135 from control and 142 from intervention). The data missing from the remaining participants could be due to failed synchronisation, use of app offline only, or not opening the app after registration.

bParticipant could select 'no aids used' or select one or more aids.

Primary and secondary outcomes. The primary outcome was self-reported abstinence in the past 14 days at 4-weeks. ${ }^{37,38}$ Analysis was as per intention to treat, and participants lost to follow up were presumed to have resumed smoking.

Secondary outcomes were (1) 6-month point prevalence (not smoking in the past 7-days) and continuous 6-month abstinence (allowing for smoking of $\leq 5$ cigarettes, and not smoking in the past 7 days) ${ }^{35}$ (2) follow-up channel; (3) app usage (logins, time spent, time/login, proportion of users accessing pre- and post-quit app, accessing craving aids); and (4) satisfaction (only 31 participants provided this data via app or email; the findings are reported in Table A.5).

\section{Data analysis}

Data analyses were conducted by the first author, with no Bupa involvement. Information on group assignment was kept separate from the primary outcome data until an analysis plan was registered on OSF (https://osf.io/vau42/). The primary outcome data were analysed by Fisher's exact test using intention-to-treat (ITT). Relative risk (RR) and 95\% confidence intervals were calculated. We also assessed abstinence at short and long term using log-binomial regressions with and without adjustment for baseline characteristics. Analyses of secondary outcomes were conducted using $t$-test and Mann-Whitney $U$-test, and chi-square. All tests were two-sided with alpha initially set to 0.05 . Sensitivity analyses were conducted $(*$ denotes pre- registered analyses) that were limited to (a) complete cases*, (b) Users Sample*, (c) Post-Quit app Users (as in SF28 analysis ${ }^{11}$ ). Sidak correction was used for multiple comparisons. Sidak was preferred over Bonferroni correction as the latter has been shown to overcorrect, unnecessarily raising the Type II error rate, while the latter is less conservative while maintaining an acceptable Type I error rate. ${ }^{39}$ We also calculated Bayes factors using an online calculator (https://medstats.github.io/bayesfactor.html). This analysis can distinguish between the likelihood of both the null and alternative hypotheses, and assess whether the data are insensitive. $^{26,30,40}$ We used a uniform distribution with an expected effect size of OR of 1 to 3 versus $1 *$. We also used a conservative approach with half-normal distribution, with the mode at 0 (no intervention effect), and the standard deviation equal to the expected effect size of $\mathrm{OR}=1.6$, and other plausible effects of $\mathrm{OR}=1.2$ and $\mathrm{OR}=2.5$.

\section{Results}

\section{Participants}

Out of 695 complete registrations, 425 participants met the inclusion criteria ( 217 were randomised to the control and 208 to the intervention; Figure 1). Participants were 33 years old on average, $45.5 \%$ were female, the majority had post-16 education and had previously used cessation 
Table 2. Abstinence rates at 4 weeks and 6.5 months in BupaQuit trial.

\begin{tabular}{|c|c|c|c|c|c|}
\hline & Int & Control & & Bayes $\mathrm{Fa}$ & $r^{a}$ distribution \\
\hline Outcome (all self-reported) & $\%(n / N)$ & $p^{1}$ & RR (95\% CI) (unadjusted) ${ }^{2}$ & Uniform & Half-normal \\
\hline
\end{tabular}

Continuous abstinence at 4-weeks

\begin{tabular}{|c|c|c|c|c|c|c|}
\hline 14-day FS, ITT & $13.5(28 / 208)$ & $15.7(34 / 217)$ & 0.58 & $0.86(0.54$ to 1.36$)$ & 0.20 & $0.64^{b}, 0.34^{c}, 0.19^{d}$ \\
\hline 14-day FS, CC & $25.7(28 / 109)$ & $28.1(34 / 121)$ & 0.77 & 0.91 (0.60 to 1.40$)$ & 0.25 & $0.73^{b}, 0.41^{c}, 0.23^{d}$ \\
\hline 14-day US, ITT & $14.1(20 / 142)$ & $16.3(22 / 135)$ & 0.62 & $0.86(0.50$ to 1.51$)$ & 0.15 & $0.47^{b}, 0.73^{c}, 0.24^{d}$ \\
\hline 14-day US, CC & $26.3(20 / 76)$ & $28.2(22 / 78)$ & 0.86 & $0.93(0.56$ to 1.56$)$ & 0.34 & $0.82^{b}, 0.52^{c}, 0.30^{d}$ \\
\hline 14-day ${ }^{\text {QU ITT }}$ & $16.5(18 / 109)$ & $19.5(22 / 113)$ & 0.60 & 0.85 (0.48 to 1.49$)$ & 0.27 & $0.73^{b}, 0.43^{c}, 0.24^{d}$ \\
\hline 14-day PQU CC & $30.0(18 / 60)$ & $32.8(22 / 67)$ & 0.85 & 0.91 (0.54 to 1.53$)$ & 0.34 & $0.81^{b}, 0.52^{c}, 0.30^{d}$ \\
\hline \multicolumn{7}{|c|}{ Abstinence at 6.5 -month } \\
\hline Sustained +, FS, ITT & $11.1(23 / 208)$ & $13.4(29 / 217)$ & 0.55 & $0.83(0.50$ to 1.38$)$ & 0.21 & $0.65^{b}, 0.35^{c}, 0.19^{d}$ \\
\hline Sustained ${ }^{\mathrm{FS}, \mathrm{CC}}$ & $29.1(23 / 79)$ & $30.4(29 / 92)$ & 0.74 & 0.92 (0.59 to 1.46$)$ & 0.29 & $0.77^{b}, 0.47^{c}, 0.27^{d}$ \\
\hline Sustained US, ITT & $10.6(15 / 142)$ & $14.1(19 / 135)$ & 0.46 & 0.75 (0.40 to 1.42$)$ & 0.23 & $0.67^{b}, 0.38^{c}, 0.21^{d}$ \\
\hline Sustained US, CC & $26.8(15 / 56)$ & $32.8(19 / 58)$ & 0.54 & 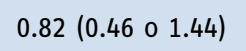 & 0.29 & $0.74^{b}, 0.45^{c}, 0.26^{d}$ \\
\hline Sustained ${ }^{\mathrm{PQU}} \mathrm{ITT}$ & $12.8(14 / 109)$ & $15.9(18 / 113)$ & 0.57 & 0.81 (0.42 to 1.54$)$ & 0.30 & $0.73^{b}, 0.44^{c}, 0.25^{d}$ \\
\hline Sustained ${ }^{\mathrm{PQU} C \mathrm{CC}}$ & $31.1(14 / 45)$ & $35.3(18 / 51)$ & 0.83 & $0.88(0.50$ to 1.56$)$ & 0.36 & $0.81^{b}, 0.54^{c}, 0.32^{d}$ \\
\hline 7-day PP FS, ITT & $14.4(30 / 208)$ & $17.1(37 / 217)$ & 0.51 & 0.85 (0.54 to 1.32$)$ & 0.18 & $0.61^{b}, 0.32^{c}, 0.17^{d}$ \\
\hline 7-day PP FS, CC & $38.0(30 / 79)$ & $40.2(37 / 92)$ & 0.88 & 0.94 (0.65 to 1.38 ) & 0.29 & $0.77^{b}, 0.46^{c}, 0.26^{d}$ \\
\hline 7-day PP US, ITT & $14.1(20 / 142)$ & $18.5(25 / 135)$ & 0.33 & 0.76 (0.44 to 1.30$)$ & 0.19 & $0.62^{b}, 0.33^{c}, 0.18^{d}$ \\
\hline 7-day PP US, CC & $35.7(20 / 56)$ & $43.1(25 / 58)$ & 0.45 & $0.83(0.52$ to 1.31$)$ & 0.26 & $0.70^{b}, 0.41^{c}, 0.23^{d}$ \\
\hline 7-day PP PQU IT & $15.6(17 / 109)$ & $21.2(24 / 113)$ & 0.30 & $0.73(0.42$ to 1.29$)$ & 0.20 & $0.62^{b}, 0.33^{c}, 0.18^{d}$ \\
\hline 7-day PP PQU CC & $37.8(17 / 45)$ & $47.1(24 / 51)$ & 0.41 & $0.80(0.50$ to 1.29$)$ & 0.26 & $0.70^{b}, 0.41^{c}, 0.42^{d}$ \\
\hline
\end{tabular}

${ }^{\mathrm{FS}}$ Full sample eligible at baseline; ${ }^{\mathrm{IT}}$ Intention-to-treat analysis; ${ }^{\mathrm{CC}}$ Complete case analysis (excluding participants who were not reached at follow-up); ${ }^{\text {US }}$ Users sample (excluding participants with app-data-missing). ${ }^{\mathrm{PQ}}$ Post-quit users (limited to participants who used the app after the quit date, when more features were available, including craving meter and craving aids).

tSustained abstinence: smoking five cigarettes or less in the past 6 months and not smoking in the past 7 days; PP: point prevalence. ${ }^{1} p$-value from Fisher's exact test.

${ }^{2}$ We conducted adjusted analyses of short- and long-term abstinence among the full study sample, which did not affect the results.

aFor Bayes factor calculation using uniform distribution (pre-registered), we set the expected effect to be between odds ratio of 1 and 3 , versus 1 . For Bayes factors calculation using the half-normal distribution (exploratory), the effect sizes used to specify the standard deviation of the theory (normal logarithm of $\mathrm{ORs}$ ) for the half-normal distributions representing the alternative hypotheses were as follows: ${ }^{\mathrm{b}} \mathrm{OR}=1.2 ;{ }^{\mathrm{c}} \mathrm{O}=1.6,{ }^{\mathrm{d}} \mathrm{OR}=2.5$ (Brown et al. 2016 ; Naughton et al. 2017). The Bayes factors presented in bold mean that the findings supported the null hypothesis, and the rest suggested the data to be insensitive.

support, and $49.3 \%$ had a manual occupation (Table 1 ). Among the trial sample, $34 \%$ classified as app-data-missing participants (i.e. they either did not access the app after registration, used it offline, or the data failed to synchronise with the server for other reasons). Except for the Users Sample being slightly older (31 vs 34 years, $p=0.01$ ), 
Table 3. Follow-up rate, follow-up channels and app usage in BupaQuit trial.

\begin{tabular}{|c|c|c|c|}
\hline & Intervention & Control & $p$ \\
\hline Follow-up rate at 4 weeks; $\%(n / N)$ & $52.4(109 / 208)$ & $55.8(121 / 217)$ & 0.49 \\
\hline \multicolumn{4}{|c|}{ Follow-up channel for primary outcome at 4 weeks; \% $(n / N)$} \\
\hline App & $7.3(8)$ & $12.4(15)$ & 0.58 \\
\hline Email & $9.2(10)$ & $9.9(12)$ & \\
\hline Phone & $80.7(88)$ & $76.0(92)$ & \\
\hline SMS & $2.8(3)$ & $1.7(2)$ & \\
\hline Follow-up rate at 6.5 months; $\%(n / N)$ & $38.0(79 / 208)$ & $42.4(92 / 217)$ & 0.35 \\
\hline Follow-up via phone at 6.5 months; \% $(n / N)$ & $77.2(61 / 79)$ & $84.8(78 / 92)$ & 0.21 \\
\hline Usage data available (during trial only); \% $(n / N)$ & $68.3(142 / 208)$ & $62.2(135 / 217)$ & 0.19 \\
\hline \multirow{2}{*}{$\begin{array}{l}\text { Total logins; Median (IQR) } \\
\text { Mean (SD) }\end{array}$} & $4.0(8.0)$ & $5.0(9.0)$ & 0.45 \\
\hline & $9.6(14.7)$ & $10.5(18.0)$ & 0.63 \\
\hline \multirow{2}{*}{$\begin{array}{l}\text { Total time }(s)^{\mathrm{b}} ; \text { Median }(I Q R) \\
\text { Mean }(S D)^{\mathrm{a}}\end{array}$} & $202.0(423.3)$ & $209.0(342.0)$ & 0.54 \\
\hline & $401.8(551.8)$ & $325.8(418.3)$ & 0.20 \\
\hline \multirow{2}{*}{$\begin{array}{l}\text { Time per login }(s)^{\text {b}} ; \text { Median (IQR) } \\
\text { Mean }(S D)^{a}\end{array}$} & $44.6(59.9)$ & $32.9(37.9)$ & 0.01 \\
\hline & $64.0(70.6)$ & $43.5(40.6)$ & 0.00 \\
\hline \multicolumn{4}{|l|}{ App usage classification ${ }^{c}$} \\
\hline Accessed only pre-quit app & $23.2(33)$ & $16.3(22)$ & 0.20 \\
\hline Accessed only post-quit app & $25.4(36)$ & $33.3(45)$ & \\
\hline Accessed both pre- and post-quit app & $51.4(73)$ & $50.4(68)$ & \\
\hline
\end{tabular}

${ }^{a}$ We provide Means to enable comparison with other studies. However, the usage data were skewed and hence we conducted and report results from non-parametric tests comparing usage between the two study arms.

${ }^{\mathrm{b}}$ Total time, excluding registration, from the first use until follow-up. The time spent is an underestimate: (a) data from offline app use save locally on user's device but would not synchronise if users had not accessed the app while being online on a future occasion; and (b) the interaction between an app page and the server occurs when a page is loaded. No further communication with the server occurs until another page is loaded. Hence, it is not possible to identify the exact duration of the last interaction when it ends with exiting the app.

'Only assessed among the sample with usage data available. Pre-quit app use only means that participants set the quit date in the future and accessed only pre-quit content; only the post-quit intervention app offered craving aids.

there was no statistically significant differences on baseline characteristics between participants with and without the app data (Table A.4).

\section{Cessation outcomes}

The overall abstinence rate was similar between the groups on the primary $(13.5 \%$ vs $15.7 \%, p=0.58)$ and secondary cessation outcomes (sustained 6-month abstinence: $11.1 \%$ vs $13.4 \%$; and 6-month point prevalence: $14.4 \%$ vs $17.1 \%$, see Table 2). The findings did not change after adjustment for baseline characteristics and in sensitivity analyses. On the primary outcome, the Bayes factor calculated using a uniform distribution supported the null hypothesis $(\mathrm{Bu}[0,0,1.0986]=0.201)$. The Bayes factors using the half-normal distribution suggested that the data were insensitive for low effect sizes, but that for $\mathrm{OR}=2.5$ the data supported the null hypothesis. Conclusions from 
Bayes factors analyses were similar for the secondary cessation outcomes (see Table 2).

\section{Follow-up rates}

At 4 -week and 6.5 -month, $54.1 \%$ and $40.2 \%$ participants were contacted, respectively, primarily via the phone (Table 3). There were no statistically significant differences in follow-up rates between the study arms, participants with or without the app data, or across baseline characteristics, except for men being more likely to be contacted at 6 months $(p=0.004)$ (data not reported).

\section{App usage}

Usage data between intervention and control participants were similar in terms of the login times (median $=4$ vs 5 , $p=0.45$; mean $=9.55$ vs $10.5, p=0.63$ ), total time spent using the app (median $=202 \mathrm{~s}$ vs $209 \mathrm{~s}, p=0.54$; mean $=$ 401.8 vs $325.8, p=0.20$ ), or the proportion of sample accessing only pre-quit content of the app $(23.2 \%$ and 16.3\%) (Table 3). Intervention users tended to spend more time on app per login (median $=44.6 \mathrm{~s}$ vs $32.9 \mathrm{~s}, p$ $=0.01 ;$ mean $=64.0 \mathrm{~s}$ vs $43.5, p=0.003$ ). Only 48 (23.1\% of all intervention participants, $44 \%$ among those using BupaQuit post-quit where craving aids were available), accessed any craving aids (median $=3$ aids accessed, range: 1-34).

\section{Discussion}

Craving management tools offered within the BupaQuit app had no detectable effect on quit rates. The self-reported quit rates were within the ranges reported in other studies $8,12,14$ and were comparable to those in SF28 study when the analysis was restricted to a similar sample of participants (users who used the app post-quit date). ${ }^{11}$ The engagement levels were comparable to SF28 app, ${ }^{11}$ but were nevertheless relatively low, including with the craving aids, which is a possible explanation for low effectiveness, which echoes findings from similar studies. ${ }^{41}$ The lack of contact with researchers at enrolment might have contributed to suboptimal engagement. ${ }^{42}$ It is also plausible that even with greater engagement, any impact of craving management tools would be too small to be detected, especially over and above the impact of other active components and evidencebased advice offered within the control app version, including setting up the quit date, monitoring of progress and using pharmacotherapy. ${ }^{43,44}$ Further factors that may have contributed to the ineffectiveness of the intervention and low engagement include a lack of in-depth co-design during the development stage, limited adaptively tailored content and exclusion of hybrid components, such as human support.
The methodological challenges encountered during the conduct of this trial point to important feasibility issues facing remote recruitment and retention in smartphonebased smoking cessation interventions, particularly when cessation apps were an emerging technology. The publication of null findings from trials of such apps is important to counter the existing in the literature biases against the null findings (i.e. the file-drawer problem) that can further hamper scientific progress. ${ }^{45}$

\section{Methodological observations}

We conducted open and automated recruitment into an RCT embedded within a cessation app that was available to anyone on UK app stores, which involved the collection of contact details. This enrolment process differed to those used in other studies that included intermediary steps of contacting the researchers or completing additional screening procedures. ${ }^{8,9,14}$ Most users who initiated registration completed baseline with all fields mandatory, and provided plausible contact details. However, policies of app stores may limit what identifiable data could be requested from users, thus affecting study procedures. Furthermore, reliance on the automated screening of app registrations emerged as insufficient. Additionally, most participants were recruited during paid advertisement campaigns. These observations suggest that dedicated budgets and human resources may be required for recruitment, enrolment, data management and follow-up in smartphone-based studies.

Nevertheless, a major challenge for conducting an evaluation of apps through randomised control design, and for recruitment into apps that are live on app stores, is that any informational and promotional materials (e.g. leaflets, but also information on app stores) must conceal the differences between the conditions. This prevents promoting many of the core features offered only within the intervention app version and may result in a recruitment campaign that is less appealing and thus likely to be less effective than one that would actively highlight the intervention features. To improve the reach of recruitment campaigns, future studies could explore diversifying promotional strategies and partnering with national or local organisations to support app promotion within their networks.

Furthermore, the telephone follow-up was the most successful, but it rarely allowed for a longer discussion with participants, while the re-contact via app, email or texting yielded very poor results. This limits the volume of secondary outcome data that can be feasibly collected in such studies. Finally, sensitivity analysis suggests that participants accessing BupaQuit app post-quit, which offered additional features (monitoring and feedback on smoking in both app versions, and craving aids in the intervention), might had higher quit rates than those who set the quit date in the future but never access the app post-quit. 
Future research should explore and account for the impact that different pre- and post-quit features in stop smoking apps might have on user behaviour and cessation.

\section{Limitations}

First, the study was underpowered to detect the original effect expected, but the Bayes factors suggest it is unlikely that a greater sample would bring support for the alternative hypothesis. Second, only a minority of participants responded to the app or e-mail follow-up, thus providing data on satisfaction. Third, despite the relatively intensive follow-up outside of the app, ${ }^{37,38,46}$ the follow-up rates were falling within the lower end of the spectrum for re-contact rates in other studies. ${ }^{8,37,47}$ The lack of incentives for participation has increased ecological validity of the findings, but it has likely negatively affected both recruitment and follow-up. ${ }^{8}$ Fourth, self-reported abstinence rates tend to overestimate the actual quit rates, although the bias may be lower in remote interventions, and should not differ across study arms. ${ }^{48,49}$ Fifth, we were missing app usage data from a third of participants who met trial eligibility criteria and could not account for this data missingness. Importantly, except for being younger, these participants did not differ from those with complete data, and excluding them from the analyses has not affected conclusions. The burden of joining this study was higher than accessing normal apps on the market but lower than that in previous studies of cessation apps. ${ }^{8,9,12}$ Nevertheless, this is limiting the generalisability of the findings to a wider population of smokers using apps.

\section{Future directions}

Managing cigarette cravings can benefit cessation, ${ }^{16,18}$ and it has been mentioned by smokers as a desired feature of digital interventions. ${ }^{50,51}$ Future research should explore new ways of delivering more engaging and usable CMTs. This could involve utilisation of user-centred approaches ${ }^{52}$ and other research designs, such as Multiphase Optimisation Strategy (MOST; ${ }^{53,54}$ ), to assess usability and impact of a range of, or a combination of, craving aids, as well as different app architectures and user journeys. It would also need to be ascertained if greater contact with researchers at enrolment could improve engagement and outcomes.

Moreover, smartphone technology and app software have rapidly progressed in the past years, paving ways for new intervention components and tools to support remote smartphone research, including hybrid approaches. These include chatbots powered by artificial intelligence to mirror human-human interaction that might be used to increase engagement with apps ${ }^{55}$ or heart rate monitoring apps that showed early promise for remote verification of abstinence from smoking. ${ }^{56}$ Such developments provide new opportunities for delivering cessation support and should be explored in relation to craving management in the future. Similarly, potential participants now may have greater experience with health-related apps, more digital skills, and different expectations for app functionality and design, which may mean different results in future studies. This is particularly true following the suspension of many face-to-face support services during the COVID-19 pandemic, which has led to service providers pivoting towards digital offers.

\section{Conclusions}

In this pragmatic trial, the addition of craving management tools to the BupaQuit app did not affect cessation, and limited engagement with the app may have contributed to this.

Acknowledgments: BupaQuit trial was conducted as part of a collaboration between Bupa and UCL. Among Bupa team (*former) we would like to especially acknowledge and thank for their support during the project: Alan Payne (Digital Director*), Lesley Payne (Head of the Global Institute for Digital Health Excellence and Senior Project Manager*), Fiona Adshead (Chief Wellbeing Officer*), Ann Sandy (Senior Project Manager*), Tuan Nguyen (Programmer*) and Ekaterina Volkova-Volkmar (Data Scientists*); as well as Dr Jenny Leeser (Clinical Director Occupational Health) and Dr Clare Scully (Director of Clinical Development) for reviewing the final manuscript and feedback. We would like to thank Bupa for offering financial support, office facilities and resources enabling conducting of the study and data collection, and Bupa's legal and internal and external communications teams for their assistance in finalising study materials and facilitating participant recruitment. We would also like to thank and acknowledge Bedfont ${ }^{\circledR}$ Scientific Ltd for providing personal CO monitoring devices for the trial, as well as for their ongoing support with $\mathrm{CO}$ personal monitors use, and for adapting the software for $\mathrm{CO}$ reading to BupaQuit trial. We are also grateful to Michael Ussher for his advice and materials on craving management activities described in BupaQuit. Finally, we would like to acknowledge the invaluable contribution of research assistants in recruitment and collection of follow-up data: Olga Perski, Courtney Kwan and Georgina Knock. AH, LS, JB, EB \& RW are part of UKCTAS.

Conflict of interest: The authors declared the following potential conflicts of interest with respect to the research, authorship and/or publication of this article: AH led the BupaQuit trial, conducted independent data analysis and prepared the manuscripts as part of her $\mathrm{PhD}$ funded by British Heart Foundation 4-year $\mathrm{PhD}$ at UCL. AH has been employed at Bupa as a Consultant (Research Partner and Expert Advisor) only during the BupaQuit app development, and Bupa had no impact on data analysis and results dissemination. $\mathrm{AH}$ has received unrestricted funds as part of a project Global Bridges at Mayo Clinic and Pfizer Independent Grants for Learning and Change Request for Proposals (RFP): EUROPEAN PROGRAM and has been acting 
as a consultant in paid and unpaid capacity on digital health, smoking cessation and behavioural science. LS has received honoraria for talks, an unrestricted research grant and travel expenses to attend meetings and workshops from Pfizer and Johnson\&Johnson, and has acted as a paid reviewer for grant awarding bodies and as a paid consultant for health care companies. Other research has been funded by the government, a community-interested company (National Centre for Smoking Cessation) and charitable sources. JB and EB have received unrestricted research grants from Pfizer. AM worked as Digital mHealth Manager at Bupa. RW undertakes research and consultancy and receives fees for speaking from companies that develop and manufacture smoking cessation medications (Pfizer, Johnson \& Johnson, GlaxoSmithKline). JB \& RW are both unpaid members of the scientific steering group of the Smoke Free mobile application. The views presented are not necessarily the views of the funders. HKU has no conflicts of interest.

Contributorship: AH conceived, set up and conducted the trial, was responsible for data analysis and wrote the first version of the manuscript. AH and AM led on the BupaQuit app day-today development and oversaw data collection and trial delivery. JB, HKU and RW consulted on app development and trial design. LS, JB, EB and RW consulted on data analysis. All authors revised the manuscript for critical content and approve its final version.

Ethical approval: The study was approved by the University College London Research Ethics Committee (ref: 6212/001).

Funding: The authors disclosed receipt of the following financial support for the research, authorship and/or publication of this article: The costs of app development and of conducting the study (including participant recruitment, data collection, the cost of purchasing $\mathrm{CO}$ monitors) were covered by Bupa. AH was leading the trial as part of her $\mathrm{PhD}$ funded by British Heart Foundation 4-year PhD Studentship at UCL [FS/13/59/30649]. Authors from UCL supported Bupa in developing BupaQuit app. Raw data from BupaQuit app was managed by Bupa. Data management and sharing with UTARG was governed by a bespoke Data Licence Agreement, according to which anonymised data from BupaQuit were shared with RW and his team for further independent processing, analysis and dissemination. In accordance with the Data License Agreement, the final manuscript was submitted to Bupa for review prior to publication to ensure that no confidential or patentable information was included in the manuscript. Authors could accept any suggestions in good faith, but were under no obligation to make any suggested changes to the manuscript. Following Bupa review no changes were made. JB \& EB salaries are funded by a programme grant from Cancer Research UK [CRUK; C1417/A22962]. EB also receives funding from the NIHR SPHR. RW's salary was funded by CRUK for part of the preparation of this manuscript. The funders had no influence over the study design, data collection, analysis, findings interpretation and preparation of the manuscript.
ORCID iD: Aleksandra Herbec (iD https://orcid.org/0000-00023339-7214

Supplemental material: Supplementary material for this article is available online on Open Science Framework at the following address: https://osf.io/7n9rp/.

\section{References}

1. Raupach T, West R and Brown J. The most "successful" method for failing to quit smoking is unassisted cessation. Nicotine Tob Res 2013; 15: 748-749.

2. Pulverman R and Yellowlees PM. Smart devices and a future of hybrid tobacco cessation programs. Telemed $J$ E Health 2014; 20: 241-245.

3. Deloitte. State of the smart. Consumer and business usage patterns. Global Mobile Consumer Survey 2017: The UK cut, 2017, http:// www.deloitte.co.uk/mobileuk/\#state-of-the-smartphone-market (2018, accessed 15 December 2018) (Archived by WebCite ${ }^{\circledR}$ at http://www.webcitation.org/74gXXcEqq).

4. Abroms LC, Lee Westmaas J, Bontemps-Jones J, et al. A content analysis of popular smartphone apps for smoking cessation. Am J Prev Med 2013; 45: 732-736.

5. Jacobs MA, Cobb CO, Abroms L, et al. Facebook apps for smoking cessation: a review of content and adherence to evidence-based guidelines. J Med Internet Res 2014; 16: e205.

6. Hoeppner BB, Hoeppner SS, Seaboyer L, et al. How smart are smartphone apps for smoking cessation? A content analysis. Nicotine Tob Res 2016; 18: 1025-1031.

7. Haskins BL, Lesperance D, Gibbons P, et al. A systematic review of smartphone applications for smoking cessation. Transl. Behav. Med 2017; 7: 292-299.

8. Bricker JB, Mull KE, Kientz JA, et al. Randomized, controlled pilot trial of a smartphone app for smoking cessation using acceptance and commitment therapy. Drug Alcohol Depend 2014; 143: 87-94.

9. Buller DB, Borland R, Bettinghaus EP, et al. Randomized trial of a smartphone mobile application compared to text messaging to support smoking cessation. Telemed $J E$ Health 2014; 20: 206-214.

10. Garrison KA, Pal P, O'Malley SS, et al. Craving to quit: a randomized controlled trial of smartphone app-based mindfulness training for smoking cessation. Nicotine Tob Res 2018; 22: 324-331.

11. Ubhi HK, Michie S, Kotz D, et al. A mobile app to aid smoking cessation: preliminary evaluation of SmokeFree28. J Med Internet Res 2015; 17: e17.

12. Bricker JB, Copeland W, Mull KE, et al. Single-arm trial of the second version of an acceptance \& commitment therapy smartphone application for smoking cessation. Drug Alcohol Depend 2017; 170: 37-42.

13. Iacoviello BM, Steinerman JR, Klein DB, et al. Clickotine, A personalized smartphone App for smoking cessation: initial evaluation. JMIR Mhealth Uhealth 2017; 5: e56.

14. BinDhim NF, McGeechan K and Trevena L. Smartphone smoking cessation application (SSC App) trial: a multicountry double-blind automated randomised controlled trial of a smoking cessation decision-aid 'app'. BMJ open 2018; 8: $\mathrm{e} 017105$. 
15. Zhou X, Nonnemaker J, Sherrill B, et al. Attempts to quit smoking and relapse: factors associated with success or failure from the ATTEMPT cohort study. Addict Behav 2009; 34: 365-373.

16. May J, Andrade J, Panabokke N, et al. Visuospatial tasks suppress craving for cigarettes. Behav Res Ther 2010; 48: 476485.

17. Ussher M, Cropley M, Playle S, et al. Effect of isometric exercise and body scanning on cigarette cravings and withdrawal 104: 1251-1257. symptoms. Addiction (Abingdon, England) 2009;

18. Haasova M, Warren FC, Ussher M, et al. The acute effects of physical activity on cigarette cravings: systematic review and meta-analysis with individual participant data. Addiction (Abingdon, England) 2013; 108: 26-37.

19. Shahab L, Sarkar BK and West R. The acute effects of yogic breathing exercises on craving and withdrawal symptoms in abstaining smokers. Psychopharmacology 2013; 225: 875-882.

20. Michie S, Hyder N, Walia A, et al. Development of a taxonomy of behaviour change techniques used in individual behavioural support for smoking cessation. Addict Behav 2011; 36: 315-319.

21. West R and Stapleton J. Clinical and public health significance of treatments to aid smoking cessation. Eur Respir Rev 2008; 17: 199-204.

22. West R and Brown J. A theory of addiction. In: West R and Brown J (eds) Theory of addiction, 2nd ed. Chichester: Wiley-Blackwell, 2013, pp. 229-256.

23. Ubhi HK, Michie S, Kotz D, et al. Characterising smoking cessation smartphone applications in terms of behaviour change techniques, engagement and ease-of-use features. Transl Behav Med 2016; 6: 410-417.

24. Michie S and West W. A guide to development and evaluation of digital behaviour change interventions in healthcare. London: Silverback Publishing, 2016, pp. 37-44.

25. West R. The clinical significance of "small" effects of smoking cessation treatments. Addiction (Abingdon, England) 2007; 102: 506-509.

26. West R. Using Bayesian analysis for hypothesis testing in addiction science. Addiction (Abingdon, England) 2016; 111: 3-4.

27. Michie S, Richardson M, Johnston M, et al. The behavior change technique taxonomy (v1) of 93 hierarchically clustered techniques: building an international consensus for the reporting of behavior change interventions. Ann Behav Med 2013; 46: 81-95.

28. Welsch SK, Smith SS, Wetter DW, et al. Development and validation of the Wisconsin smoking withdrawal scale. Exp Clin Psychopharmacol 1999; 7: 354-361.

29. Ploderer B, Smith W, Pearce J, et al. A mobile app offering distractions and tips to cope with cigarette craving: a qualitative study. JMIR Mhealth Uhealth 2014; 2: e23.

30. Brown J, Michie S, Walmsley M, et al. An online documentary film to motivate quit attempts Among smokers in the general population (4Weeks2Freedom): a randomized controlled trial. Nicotine Tob Res 2016; 18: 1093-1100.

31. Cropley M, Ussher M and Charitou E. Acute effects of a guided relaxation routine (body scan) on tobacco withdrawal symptoms and cravings in abstinent smokers. Addiction (Abingdon, England) 2007; 102: 989-993.
32. Tang YY, Tang R and Posner MI. Brief meditation training induces smoking reduction. Proc Natl Acad Sci USA 2013; 110: 13971-13975.

33. Scerbo F, Faulkner G, Taylor A, et al. Effects of exercise on cravings to smoke: the role of exercise intensity and cortisol. J. Sports Sci 2010; 28: 11-19.

34. Ussher M, Aveyard P, Manyonda I, et al. Physical activity as an aid to smoking cessation during pregnancy (LEAP) trial: study protocol for a randomized controlled trial. Trials 2012; 13: 186.

35. West R, Hajek P, Stead L, et al. Outcome criteria in smoking cessation trials: proposal for a common standard. Addiction (Abingdon, England) 2005; 100: 299-303.

36. Herbec A, Brown J, Shahab L, et al. Lessons learned from unsuccessful use of using personal carbon monoxide monitors to remotely assess abstinence in a pragmatic trial of a smartphone stop smoking app - A secondary analysis. Addict Behav Rep 2018; 13: 100333.

37. Brown J, Michie S, Geraghty AWA, et al. Internet-based intervention for smoking cessation (StopAdvisor) in people with low and high socioeconomic status: a randomised controlled trial. Lancet Respir Med 2014; 2: 997-1006.

38. Herbec A, Brown J, Tombor I, et al. Pilot randomized controlled trial of an internet-based smoking cessation intervention for pregnant smokers ('MumsQuit'). Drug Alcohol Depend 2014; 140: 130-136.

39. Holland BS and Copenhaver MD. Improved Bonferroni-type multiple testing procedures. Psychol Bull 1988; 104: 145-149.

40. Dienes Z. Using Bayes to get the most out of non-significant results. Front Psychol 2014; 5: 781.

41. Shahab L and McEwen A. Online support for smoking cessation: a systematic review of the literature. Addiction (Abingdon, England) 2009; 104: 1792-1804.

42. Perski O, Blandford A, West R, et al. Conceptualising engagement with digital behaviour change interventions: a systematic review using principles from critical interpretive synthesis. Transl Behav Med 2017; 7: 254-267.

43. West R, Walia A, Hyder N, et al. Behavior change techniques used by the English stop smoking services and their associations with short-term quit outcomes. Nicotine Tob Res 2010; 12: 742-747.

44. Lorencatto F, West R and Michie S. Specifying evidencebased behavior change techniques to aid smoking cessation in pregnancy. Nicotine Tob Res 2012; 14: 1019-1026.

45. Munafo M and Neill J. Null is beautiful: on the importance of publishing null results. $J$ Psychopharmacol (Oxford, England) 2016; 30: 585.

46. Free C, Knight R, Robertson S, et al. Smoking cessation support delivered via mobile phone text messaging (txt2stop): a single-blind, randomised trial. Lancet 2011; 378: 49-55.

47. Taylor GMJ, Dalili MN, Semwal M, et al. Internet-based interventions for smoking cessation. Cochrane Datab System Rev (Online) 2017; 9: CD007078.

48. Glasgow RE, Mullooly JP, Vogt TM, et al. Biochemical validation of Smoking status: pros, cons, and data from four lowintensity intervention trials. Addict Behav 1993; 18: 511-527.

49. Patrick DL, Cheadle A, Thompson DC, et al. The validity of self-reported smoking: a review and meta-analysis. Am J Public Health 1994; 84: 1086-1093.

50. Herbec A, Beard E, Brown J, et al. The needs and preferences of pregnant smokers regarding tailored internet-based 
smoking cessation interventions: a qualitative interview study. BMC Public Health 2014; 14: 1070.

51. Herbec A, Perski O, Shahab L, et al. Smokers' views on personal carbon monoxide monitors, associated apps, and their Use: an interview and think-aloud study. Int $J$ Environ Res Public Health 2018; 15: E288.

52. Yardley L, Morrison L, Bradbury K, et al. The person-based approach to intervention development: application to digital health-related behavior change interventions. $J$ Med Internet Res 2015; 17: e30.

53. Collins LM, Dziak JJ, Kugler KC, et al. Factorial experiments: efficient tools for evaluation of intervention components. Am J Prev Med 2014; 47: 498-504.
54. Collins LM, Murphy SA and Strecher V. The multiphase optimization strategy (MOST) and the sequential multiple assignment randomized trial (SMART): new methods for more potent eHealth interventions. Am J Prev Med 2007; 32: S112-S118.

55. Perski O, Crane D, Beard E, et al. Does the addition of a supportive chatbot promote user engagement with a smoking cessation app? An experimental study. Digit Health 2019; 5: 2055207619880676.

56. Herbec A, Parker E, Ubhi HK, et al. Decrease in resting heart rate measured using smartphone apps to verify abstinence From smoking: an exploratory study. Nicotine Tob Res 2020; 22: 1424-1427. 